\title{
SOME IMPORTANT APPLICATIONS OF SEMIGROUPS
}

\author{
${ }^{1}$ Iqra Liaqat $\&{ }^{2}$ Wajeeha Younas \\ ${ }^{1,2}$ Department of Mathematics, Punjab University, New Campus, \\ Lahore District-75500, Pakistan
}

Email:pro.iqra.14@gmail.com

\begin{abstract}
This Paper deals with the some important applications of semigroups in general and regular semigroups in particular.The theory of finite semigroups has been of particular importance in theoretical computer science since the 1950s because of the natural link between finite semigroups and finite automata via the syntactic monoid. In probability theory, semigroups are associated with Markov process. In section 2 we have seen different areas of applications of semigroups. We identified some Applications in biology, Partial Differential equation, Formal Languages etc whose semigroup structures are nothing but regular.
\end{abstract}

Keywords. Semigroups of Operators, Abstract Cauchy problem, Coagulation.

\section{Introduction.}

Semigroups are very natural and general structures and enter our mathematical life from the very beginning ( $\mathbb{N}$ with respect to addition, multiplication, maximum or minimum, sets with respect to union or intersection). Due to their simple axioms they are very often and easily found. If $\mathrm{M}$ is a nonempty set and $S=M^{M}$ is the set of all mappings from $M$ to $M$, then $S$ is a semigroup with respect to composition, and in fact every semigroup can be realized as a subsemigroup of $\mathrm{M}^{\mathrm{M}}$ from M.

The class of semigroups being so vast it might seem reasonable to limit somehow this generality. We shall do this in a very specific way by looking primarily to abelian semigroups. On the other hand, in many situations the semigroups we meet carry a natural involution, i.e. a mapping -: $S \rightarrow$ $\mathrm{S}$ such that $(\mathrm{st})^{-}=\mathrm{t}^{-} \mathrm{s}^{-}$and $\left(\mathrm{s}^{-}\right)^{-}=\mathrm{s}$. On a group we always have the group involution $\mathrm{s}^{-}=\mathrm{s}^{-1}$. As another example (used later on) consider the upper half -plane with usual addition and

$$
(x, y)^{-}:=(-x, y)
$$

Contrary to the group case, the existence of a neutral element is not automatic in semigroups. Although there are important instances where this non-existence is natural, we will - if not explicity told otherwise - tacitly assume that the semigroups considered do have a neutral element. So a semigroup for us will be a triple $S=(S,+,)^{\prime}$ where $S$ is a (nonempty) set, "' + ', is a commutative semigroup operation, and " $-"$ " is an involution on $\mathrm{S}$ (very often "= id in ehich case we simply write $S=(S,+)$ ). The neutral element will be denoted by ' 0 ', 
The simplest functions on a semigroup 'respecting' the semigroup structure are certainly the scalar - valued homomorphisms, i.e. the characters. They are the building stones for many other important function families, such as positive or negative definite functions, and some related classes as well. An excellent survey on this topic from an analytical point of view has recently been given by C. Berg [1] and is strongly recommended as a supplement to the present paper.

When mentioning semigroups in connection with probability theory, the first thought perhaps goes to one -parameter semigroups and infinitely divisible distributions. This is certainly an important area of research and of beautiful results, but it is by no means the only nontrivial occurrence of semigroups, as will be demonstrated in the sequel. Notions from Harmonic Analysis on semigroups play a dominant (and sometimes perhaps unexpected) role here, too.

Semigroups can be used in biology to describe certain aspects in the crossing of organisms, in genetics and in consideration of metabolisms. The growth of plants can be described algebraically in Hermann and Rosenberg (1975). Further material on this subject is contained in Holcombe (1982). Details on the use of semiautomata in metabolic pathways and the aid of a computer therein, including a theory of scientific experiments, can be found in Krohn, Langer and Rhodes (1976). Rosen (1973) studies ways in which environmental changes can affect the repair capacity of biological systems and considers carcinogenesis and reversibility problems. Language theory is used in cell-development problems, as introduced by Lindenmayes (1968), Hermann and Roscendalg (1975). Suppose (1969) Kiesras (1976) develop a theory of learning in which a subject is instructed to behave like a semiautomaton.

\section{Semigroups and its important Applications}

Now a days the theory of semigroups has been expanded greatly due to its applications to computer science and we also finds its usage in biology science and Partial Differential Equation. In this section we discuss some important applications of semigroups in different areas.

\section{Automaton-Semigroups.}

A finite-state machine (FSM) or finite-state automaton (FSA, plural:automata), finite automaton, or simply a state machine, is a mathematical model of computation. It is an abstract machine that can be in exactly one of a finite number of states at any given time. The FSM can change from one state to another in response to some inputs; the change from one state to another is called transition. An FSM is defined by a list of its states, its initial state , and the inputs that trigger each transition. Finite - state machines are of two types deterministic finite-state machines and non-deterministic finite-state machines. A deterministic finite-state machine can be constructed equivalent to any non-deterministic one.

The behavior of state machines can be observed in many devices in modern society that perform a predetermined sequence of actions depending on a sequence of events with ehich they are presented. Simple examples are vending machines, which dispense products when the proper 
combination of coins is deposited elevators, whose sequence of stops is determined by the floors requested by riders, traffic lights. Which change sequence when cars are waiting, and combination locks. Which require the input of a sequence of numbers in the proper order.

The finite-state machine has less computational power than some other models of computation such as the Turing machine. The computational power distinction means there are aomputational tasks that a Turing machine can do but an FSM cannot. This is because an FSM's memory is limited by the number of states it has FSM's are studied in the more general field of automata theory.

\section{Automata:}

Automata have been defined as models of systems reacting upon sequences of stimuli. Computers are among the most common examples of automata. Living organisms, many types of machines, information transmission channels, complex systems as e.g. traffic are further typical examples.

An automaton A consists of

(a) the set $X \neq \varphi$ of elementary stimuli, called input symbols,

(b) the memory S, considered as a nonempty set, the set of states, which may have an algebraical or topological structure,

(c) the set $Y \neq \varphi$ of elementary reactions, called output symbols,

(d) the function $\delta: X \times S \rightarrow S \times Y$, which to each input symbol $\mathrm{x} \in \mathrm{X}$ and each state $\mathrm{s} \in \mathrm{S}$ associaties by $\delta(\mathrm{x}, \mathrm{s})=\left(\mathrm{s}^{\prime}, \mathrm{y}\right)$ the next state $\mathrm{s}^{\prime}$ and the corresponding output symbol $\mathrm{y}$.

\section{Semigroups - Formal Languages.}

Besides the "'natural', languages such as English, German , French or Chinese, the so-called formal languages are of importance in the formal sciences, especially in computing or information sciences. The underlying principle is always the same: Given an 'alphabet' or "vocabulary' ( consisting of letters, words, punctuation symbols, number symbols, programming instructions ,etc.) one has a method ("grammar') for constructing 'meaningful'" words or sentences (i.e. the ' 'language') from this alphabet. This immediately reminds us of the term '"word semigroup', and , indeed, these free semigroups will play a major role, the language $\mathrm{L}$ constructed will be a subset of the free semigroup $\mathrm{F}_{\mathrm{A}}$ on the alphabet $\mathrm{A}$.

There are essentially three ways to construct a language L.

(a) Approach via grammar: given a collection rules ("grammar"), generate L from .

(b) Approach via automata: consider an initial semiautomaton which processes the elements of $\mathrm{L}$ in a suitable way. 
(c) Algebraic approach: $\mathrm{L}$ is constructed by the algerbraic combination of certain subsets of $\mathrm{F}_{\mathrm{A}}$.

In all three approaches we shall use algebraic methods. First we study the different approaches, then investigate connections between them. The whole theory is part of mathematical linguistics, which also uses probabilistic methods. We use the following notation : $A *$ is the free semigroup over a set $A, A^{*}$ is the free monoid over $A$, so $A^{*}=A * U\{\Lambda\}$, where $\Lambda$ denotes the empty word.

\section{Semigroups in Biology.}

The general approach that allows to construct the Markov processes describing various processes in mathematical biology (or in other applied sciences) is presented. The Markov processes are of a jump type and the starting point is the related linear equations. They describe at the micro-scale level the behavior of a large number $\mathrm{N}$ of interacting individuals (entities). The large individual limit

$\left({ }^{\prime \prime} \mathrm{N} \rightarrow \infty^{\prime \prime}\right)$ is studied and the intermediate level (the meso-scale level) is given in terms of nonlinear kinetic-type equations. Finally the corresponding systems of nonlinear ODEs (or PDEs) at the macroscopic level (in terms of densities of the interacting subpopulations) are obtained. Mathematical relationships between these three possible descriptions are presented and explicit error estimates are given. The general framework is applied to propose the microscopic and mesoscopic models that correspond to well-known systems of nonlinear equations in biomathematics.

\section{Semigroups in Probability Theory.}

One of the most fundamental operations im probability theory is convolution, and a large part of classical probability theory deals with $\mathrm{M}_{+}{ }^{1}(\mathbb{R})$, the probability distributions on the real line, being a (commutative) semigroup with respect to convolution and carrying the natural involution $\mu^{-}$ (B) $:=\mu(-B)$. Two basic procedures of obtaining new distributions from given ones are finite convolution products and limits of such finite convolutions (in the weak topology). Both these operations are in general difficult to perform by direct calculation. As an aid of great importance we have the classical Fourier transformation

$$
\begin{aligned}
\mathrm{F}: \mathrm{M}_{+}{ }^{1}(\mathbb{R}) & \rightarrow \mathrm{C}^{\mathrm{R}} \\
\mu & \mapsto\left(\mathrm{t} \mapsto \int \mathrm{e}^{\mathrm{itx}} \mathrm{d} \mu(\mathrm{x})\right)
\end{aligned}
$$

being a semigroup isomorphism and also a homeomorphism onto its images, if we consider $\mathrm{C}^{\mathrm{R}}$ with pointwise operations.

\section{Semigroups in Partial differential equations.}

Semigroup theory can be used to study some problems in the field of partial differential equations. Roughly speaking, the semigroup approach is to regard a time-dependent partial differential 
equation as an ordinary differential equation on a function space. For example, consider the following initial/ boundary value problem for the heat equation on the spatial interval $(0,1) \subset \mathrm{R}$ and times $\mathrm{t} \geq 0$.

$$
\left\{\begin{array}{l}
\partial t u(t, x)=\partial x u(t, x), x \in(0,1), t>0 \\
u(t, x)=0, x \in\{0,1\}, t>0 \\
u(t . x)=u(x), x \in(0,1), t=0
\end{array}\right.
$$

Let $\mathrm{X}=\mathrm{L}^{2}((0,1) R)$ be the $\mathrm{L}^{\mathrm{p}}$ space of square -integrable real-valued functions with domain the interval $(0,1)$ and let $A$ be the second -derivative operator with domain $D(A)=\{u \in$ $\left.\mathrm{H}^{2}((0,1) ; \mathrm{R}) \mid \mathrm{u}(0)=\mathrm{u}(1)=0\right\}$,

where $\mathrm{H}^{2}$ is a Sobolev space. Then the above initial/boundary value problem can be interpreted as an initial value problem for an ordinary differential equation on the space $\mathrm{X}$.

$$
\left\{\begin{array}{l}
u(t)=A u(t) \\
u(0)=u
\end{array}\right.
$$

On an heuristic level, the solution to this problem "'ought'" to be $u(t)=\exp (t A) u_{0}$. However , for a rigorous treatment, a meaning must be given to the exponential of tA. As a function of $t$, $\exp (\mathrm{tA})$ is a semigroup of operators from $\mathrm{X}$ to itself, taking the initial state $\mathrm{u}_{0}$ at time $\mathrm{t}=0$ to the state $u(t)=\exp (t A) u_{0}$ at time $t$. The operator $A$ is said to be the infinitesimal.

\section{References}

1. Arbib, M.A. "Algeraic theory of machines, Languages and Semigroups" New York (1968), Academic press.

2. Arbib, M.A. 'Theories of Abstract Automata", Englewood Cliffs, N.J.: Prentice Hall .(1969)

3. Boorman, S.A. and White, H.C. 'Social structures from multiple networks-II: role Structures." Amer.J.Sociol.81(1976), 1384-1466.

4. Boyd, J.P., Haeh1,J.H. and Sailer, L.D. 'Kinship Systems and Inverse semigroups", J.Math.Social.76,(1972), 37-61.

5. Clifford, A.H. and preston, G.B. 'The algebraic theory of semigroups ', Math.Surveys No.7, Amer.math.,soc., Vol.I,(1967).

6. Clifford, A.H. and preston, G.B . 'The algebraic theory of semigroups', Math . Surveys No.7, Amer .math.soc., Vol.II, (1967).

7. M. Lachowicz, Individually-based Markov processes modeling nonlinear systems in mathematical biology, Nonlinear Analysis Real World Appl., 12(4), 2396-2407 (2011) .

8. M. Lachowicz and T. Raybukha, Equilibrium solution for microscopic stochastic system in Population Biology, Math .Bisci. Engin., 10,777-786 (2013).

9. Berg, C., J.P.R. Christensen and P. Ressel (1984). Harmonic Analysis on New Semigroups. Graduate Texts in Mathematics, Vol. 100. Berlin - Heidelberg-New York Springer -Verlag.

10. Bisgaard, T.M. (1990). Hoeffding's inequalities: A counterexample. J. Theoretical Probability 3 , 71-80. 